\title{
SPIDERS (ARANEAE) OF SELECTED SINKHOLES OF MORAVSKÝ KRAS PROTECTED LANDSCAPE AREA (CZECH REPUBLIC)
}

\author{
V. Hula, O. Košulič, P. Štastná
}

Received: August 26, 2011

\begin{abstract}
HULA V., KOŠULIČ O., ŠŤASTNÁ P.: Spiders (Araneae) of selected sinkholes of Moravský kras Protected Landscape Area (Czech Republic). Acta univ. agric. et silvic. Mendel. Brun., 2012, LX, No. 1, pp. 57-70

In this study, we present faunistic data about spiders in selected sinkholes of northern part of Moravský kras Protected Landscape Area. Time of collection was established in the following terms: 24 March 2010 - 22 September 2010. We collected altogether 5742 adult specimens which were determined to 59 species of 14 families. We found two very rare spiders (critically endangered Porrhomma errans and endangered Walckenaeria monoceros) and several interesting, rarely collected bioindicator species (Alopecosa trabalis, Mecopisthes silus, Zelotes longipes). From the bioindicative evaluation point of view, $44 \%$ of found species belong to species with connection to natural habitats, $37 \%$ belong to species preferring semi-natural habitats, and 19\% belong to species of disturbed habitats. From the relictness point of view, majority of species was of the expansive category (53\%), $40 \%$ of class II relicts, and only $7 \%$ of class I relicts. Sink holes did not increase total biodiversity of agricultural land too much because of their relative small size.
\end{abstract}

Araneae, faunistics, sinkholes, Moravský kras PLA

Nature in central Europe is under a very strong pressure. Over the last 100 years, there have been very significant changes in the landscape use. One of them was the connection of field plots to large areas. This caused a loss of landscape heterogeneity and a significant loss of biodiversity. In fact, there is no diversity in current agrarian landscape. Heterogeneity is represented only by small pieces of wood in the middle of fields and, in some places, also by catch crop incurred within the EU subsidies. Unfortunately, we do not know how big the landscape heterogeneity should be there in order to grow the diversity back.

In Moravský kras PLA there is a huge range of natural biotopes, however, most of them are located in the southernmost part (Hády, Ochoz, Lysá hora) or in the karst valleys. Agrarian landscape is relatively homogeneous, it is located mainly in the karst plateaus. In past years, in particular in 70s-80s, these plateaus (Ostrovská plošina a Harbechy) were converted made into arable land. Sinkholes are ones of the few elements of this landscape, which increase the heterogeneity of the environment. They are mostly without management (or were without management for a long time). Some of the sinkholes were systematically destroyed - filled by soil or in some cases by waste. Nowadayws, the entire plateau is protected and, thanks to the agro-environmental subsidies from Ministry of Agriculture of the Czech Republic, most of the Ostrovská plošina surface is covered by grass. Interest in sinkholes has also increased currently. Previously, they represented neglected areas, but recently they have become important places for wintering bats (usually open entrances to caves). We therefore wondered whether there are some interesting species in these places and how big are their populations. Fauna of sinkholes in the Czech Republic was studied only by Horáková (2005) and Horáková et al. (2005), no more works exist. 


\section{MATERIALS AND METHODS}

Spiders were collected at selected locations according to the methodology proposed by Řezáč (2009): formaldehyde pitfall traps with roofs. The pitfall traps were installed on 24 March 2010. Nine traps were placed over the entire sinkhole. Two traps (the first one and the last one) were placed outside the sinkhole in surrounding vegetation, four traps were placed on the sides of the sinkhole (in the upper part and in the slope) and one was placed at the bottom of the sinkhole (if possible because sinkhole). In this design (Fig. 1), traps were placed in all the monitored areas (7 sinkholes, Fig. 2), thus a total number of 63 traps were placed. Traps lines were always placed in the north-south orientation. Traps were collected at regular monthly intervals from April to September 2010 (22 April, 19 May, 28 June, 22 July, 26 August, and 22 September). A 4\% formaldehyde with a detergent addition as a wetting agent was used as fixative fluid. After collection, the obtained material was preserved in $70 \%$ alcohol.

The collected material was evaluated by using of approach of species relictness according to Buchar (1983) who classified spider species into groups according to their relationship to originality

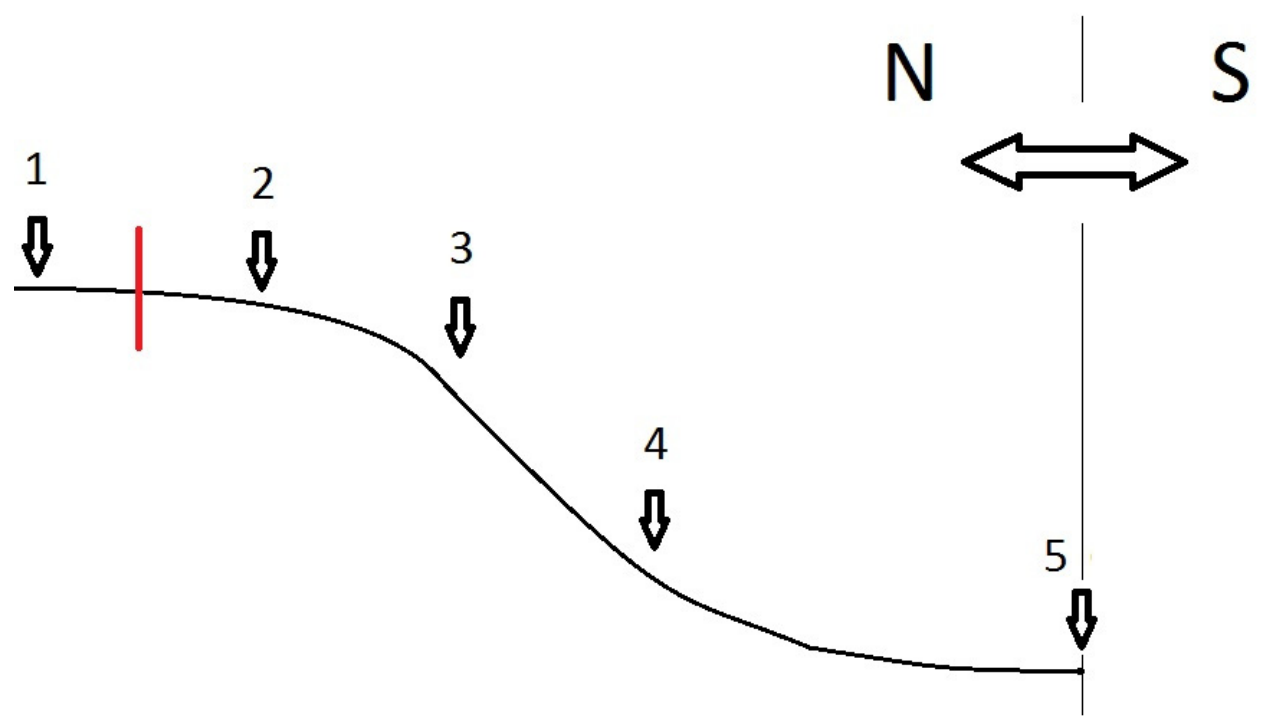

1: Design of placement of particular traps (half of sinkhole)

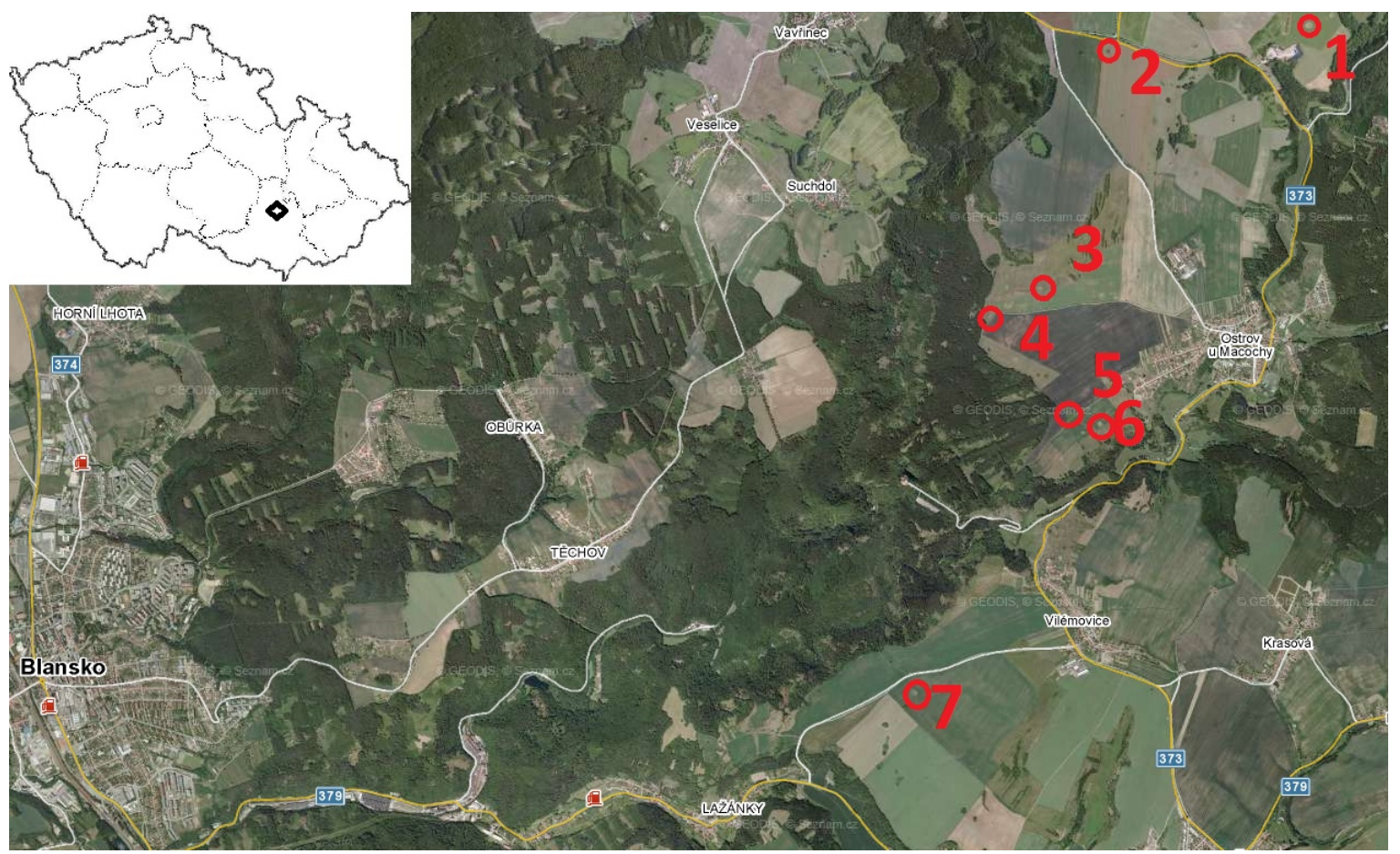

2: Map of particular sinkholes (source: www.mapy.cz) 
(disturbance) of habitats into 3 groups (RI - relict of the first class, RII - relict of the second class, E - expansive species). Another evaluation was conducted according to the methodology proposed by Buchar \& Růžička (2002), later supplemented with a further work by Růžička \& Buchar (2008), and edited by Řezáč (2009). These authors defined four categories of biotopes according to indicating classification into the following groups of spiders: climax communities (C), secondary, semi-natural habitats (SN), regularly disturbed habitats (D) and artificial habitats of human settlements (A). Thermopreference assesment of the species is based on Buchar \& Růžička (2002) who applied division of our arachnofauna into thermophilic (T), mesophilic $(\mathrm{M})$, and psychrophilic $(\mathrm{O})$ species.

Nomenclature and arrangement of families, genera and species follow the Catalogue of spiders of the Czech Republic (Buchar \& Růžička, 2002), and the most recent version of The World Spider Catalogue 12.0 (Platnick, 2011), with extention of name Clerck, where we used recommendation (Art 3.1) of ICZN (1999). Material was determined by Ondřej Košulič and Vladimír Hula, and is deposited in collection of Ondřej Košulič.

\section{Description of studied sinkholes}

Sinkhole 1: Located in permanent grasslands, meadow vegetation reflected former field crops. The center of the sinkhole was covered by nitrophilous vegetation species, especially by nettles. During the year, two mowing destroyed some pitfall traps or their roofs. GPS: $49^{\circ} 24^{\prime} \mathrm{N}, 16^{\circ} 46^{\prime} \mathrm{E}$;

Sinkhole 2: Located in field crops (2010 winter cereal), ruderal and strongly nitrophilous vegetation in the center. GPS: $49^{\circ} 24^{\prime} \mathrm{N}, 16^{\circ} 44^{\prime} \mathrm{E}$;

Sinkhole 3: Located in permanent grasslands. The entire sinkhole was heavily overgrown by nitrophilous species, especially by sorrel (Rumex spp.). GPS: $49^{\circ} 23^{\prime} \mathrm{N}, 16^{\circ} 44^{\prime} \mathrm{E}$;

Sinkhole 4: Located in permanent grasslands. The very species-rich vegetation composition of surrounding permanent grasslands was very close to potential natural grassland habitats in the area. There were indeed nitrophilous species present in the sinkhole. However, their amount was the lowest in comparison with those of the rest of monitored sinkholes. GPS: 49² $23^{\prime} \mathrm{N}, 16^{\circ} 44^{\prime} \mathrm{E}$;

Sinkhole 5: With fewer nitrophilous species as well; its surrounding corresponded to cultural meadow without special mowing. GPS: $49^{\circ} 22^{\prime} \mathrm{N}$, $16^{\circ} 44^{\prime} \mathrm{E}$;

Sinkhole 6: Located in field crops (2010 cereal), the center of sinkhole was strongly degraded due to a large number of nitrophilous species and heavy flooding during torrential rains. GPS: $49^{\circ} 22$ 'N, $16^{\circ} 44^{\prime} \mathrm{E}$;

Sinkhole 7: In 2010, it was surrounded by field crops (opium poppy), but the sinkhole was in very bad condition due to torrential rains. The sinkhole was covered by cultural meadow vegetation with great representation of dicotyledonous species and relatively small representation of ruderal nitrophilous species. GPS: $49^{\circ} 21^{\prime} \mathrm{N}, 16^{\circ} 43^{\prime} \mathrm{E}$.

\section{RESULTS}

During the arachnological research of sinkholes area from 24 March 2010 to 22 September 2010, a total number of 5742 adult spiders were collected. Out of this number, 59 species belonging to 14 families were determined. In addition, 687 juvenile specimens were found. Three expansive species of the family Lycosidae (Pardosa amentata, P. palustris, and P. pullata) were the most numerous representatives. The first two above-mentioned species comprised nearly half of all of the collected material. The highest occurrence of spiders was recorded in the period of 24 March-19 May 2010 when other representatives, such as Alopecosa cuneata, A. pulverulenta, Pachygnatha degeeri, and Trochosa terricola, reached high abundances as well. Two rare species listed in the Red List of endangered animals (Růžička, 2005) were found - critically endangered Porrhomma errans and endangered Walckenaeria monoceros. Among other relicts, two typical thermophilic species (Alopecosa trabalis [RII] and Zelotes longipes [RI]) were observed relatively frequently. Mecopisthes silus (RI), which lives typically in preserved peat bogs, spruce, and waterlogged beechwood (Buchar \& Růžička, 2002), appears to be an interesting finding as well.

According to the thermopreference assesment, the highest number of species belongs to mesophilic (55\%) and thermophilic (30\%) class. Species living mainly in colder areas of oreophyticum (psychophilic class) are represented by the remaining 15\%. In the Moravský kras PLA, thermophilnous species were found mainly in the southern part towards the massif of Hády. The biotope gradually changes into mesophyticum to the north of the studied sinkholes. That was evident as more than half of representations consisted of mesophilic species. Solely thermophilic species were represented by Porrhomma errans and Walckenaeria monoceros which are among the most important findings in the surveyed area.

According to the bioindicative evaluation (Buchar \& Růžička, 2002; Růžička \& Buchar, 2008; Řezáč, 2009), 44\% species preferred original habitats and $37 \%$ preferred semi-natural habitats. The remaining 19\% species also settled in habitats with high level of disturbance. It should be noted that members of the latter class dominated numerically in the material collected from the studied sinkholes. On the other hand, there were very few (often only one adult specimen) species preferring natural undisturbed habitats. Therefore, it is better to use assessment according to species relictness (Buchar, 1983) instaed of classifying C, SN, D and A. In this assessment, only four RI species (7\%) and 24 RII species (40\%) were found. These species inhabited natural and semi-natural habitats represented here by forest habitats. Most of the RII specimens were 
discovered in the fourth and fifth sinkholes. These sinkholes were located nearby a forest habitat where these species could come from. The remaining part of the material (31 species, 53\%) belonged to expansive species that inhabit disturbed ruderal habitats and survived here successfully. This group is represented by the dominant species of the material that occurred in high numbers in most of the investigated sinkholes.

\section{Annotated list of species found}

Distribution and ecology of species follow Buchar \& Růžička (2002), Růžička \& Buchar (2008), and other literature cited under particular species. The categories of threat follow Růžička (2005): CR (critically endangered), EN (endangered). All dates are in the central European format of dd.m.yyyy.

\section{Mimetidae}

Ero furcata (Villers, 1789)

The most abundant representative of the pirate spiders having a continuous occurrence throughout the Czech Republic. Very abundant in various open as well as in forest habitats.

Data: 1ठ, 24. 3.-22. 4. 2010, sinkhole 2.

\section{Linyphiidae}

Bathyphantes nigrinus (Westring, 1851)

Very abundant species of wet biotopes such as waterlogged meadows, floodplain forests, waterlogged alder and waterways banks.

Data: 1, 24. 3.-22. 4.2010, sinkhole 7.

Centromerita bicolor (Blackwall, 1833)

Very abundant species in natural as well as in ruderal, human disturbed habitats like fields, dumps, heap dumps, and cultural meadows.

Data: 2 ㅇ, 24. 3.-22. 4. 2010, sinkhole 2; 1 우 24. 3.22. 4. 2010, sinkhole 6 .

Centromerus sylvaticus (Blackwall, 1841)

Common species on different types of open and forest habitats.

Data: 5 ㅇ, 3ㅅ, 24. 3.-22. 4. 2010, sinkhole 2; 1 , 24. 3.-22. 4. 2010; 2 , 28. 6.-22. 7. 2010, sinkhole 4; 2o, 24. 3.-22. 7. 2010; 3ㅇ, 19. 5.-28. 6. 2010 sinkhole 6.

Diplocephalus cristatus (Blackwall, 1833)

Very abundant species of open habitats, often in fields and other ruderal habitats.

Data: 1ㅇ, 24. 3.-22. 4. 2010, sinkhole 2; 4ㅇ , 22. 4.19. 5. 2010, sinkhole 4 .

Diplocephalus picinus (Blackwall, 1841)

Very abundant species primarily in wet habitats.

Data: 2 , , 24. 3.-22. 4. 2010, sinkhole 3; 8 ㅇ, 10, 24. 3.-22. 4. 2010, sinkhole 4; 4ㅇ , 24. 3.-22. 4. 2010; 1이 19. 5.-28. 6. 2010, sinkhole 6; 4ㅇ, 24. 3.-22. 4. 2010; 1ㅇ, 22. 4.-19. 5. 2010, sinkhole 7.

Diplostyla concolor (Wider, 1834)

Very abundant species occurring in many different habitats. The highest abundances are reached in floodplain forests, smaller number can be found on moist meadows and fields.

Data: 1 ㅇ, 1ð, 24. 3.-22. 4. 2010, sinkhole 2; 1 우, 24. 3.-22. 4. 2010, sinkhole 3; 3, 10ิ, 24. 3.-22. 4. 2010, sinkhole 4; 2 ㅇ 19. 5.-28. 6. 2010, sinkhole 6; 2ㅇ, 10, 24. 3.-22. 4. 2010, sinkhole 7 .

Erigone atra Blackwall, 1833

Abundant species of small spiders typical for various open unshaded habitats such as meadows, fields, and pastures, where it reaches high abundances.

Data: 9, 22. 4.-19. 5. 2010, 9 , 19. 5.-26. 8. 2010; 2 , , 28. 6.-22. 7. 2010, sinkhole 1; 4q, 2ิે, 24. 3.22. 4. 2010, 2 9 , 22. 4.-19. 5. 2010, 2 ㅇ, 19. 5.-28. 6. 2010, sinkhole 2 ; 2 , 24. 3.-22. 4. 2010, 5 \% , 26. 8.22. 7. 2010, sinkhole 3; 2 ㅇ, 24. 3.-22. 4. 2010, 4우, 22. 4.-19. 5. 2010, sinkhole 4; 19, 28. 6.-22. 7. 2010, sinkhole 5; 17ㅇ, 24. 3.-22. 4. 2010, 7ㅇ, 22. 4.-19. 5. 2010, 2 ㅇ, 19. 5.-28. 6. 2010, sinkhole 6; 35우, 8ㅅ, 24. 3.-22. 4. 2010, 6ㅇ , 22. 4.-19. 5. 2010, 2 ㅇ, 22. 7.26. 8. 2010, sinkhole 7 .

\section{Maso sundevalli (Westring, 1851)}

Common species living in the underwood of all types of forest ecosystems.

Data: 1ㅇ, 28. 6.-22. 7. 2010, sinkhole 3; 1ㅇ, $2 \hat{\jmath}$, 22. 4.-19. 5. 2010, sinkhole 5; 19, 24. 3.-22. 4. 2010, sinkhole 6; 1ㅇ, 24. 3.-22. 4. 2010, sinkhole 7.

Mecopisthes silus (O. P.-Cambridge, 1872)

Rare species of undisturbed habitats such as peat bogs, waterlogged spruce and beech forests. Numerous findings are identified from the hill of Stožec in Šumava (Kůrka, 1982) and Rejvíz in Hrubý Jeseník (Miller, 1951). From the area of South Moravia, a finding was reported by prof. F. Miller from Pouzdrany NNR - Kolby in the 60s of the last century (Bryja et al., 2005). To date, occurrence of this species in southern Moravia has not been confirmed. In the nearby surrounding of the surveyed sinkholes, a finding from the slopes of Vilémovická and Macošská stráň has been reported (Niedobová et al., 2011).

Data: 1ㅇ, 28. 6.-22. 7. 2010, sinkhole 3.

Meioneta rurestris (C. L. Koch, 1836)

Abundant epigeic species, ubiquitous aeronaut in various sites in the forest and outside it, in agrobiocenoses and synanthropicly on the walls of houses.

Data: 1, 24. 3.-22. 4. 2010, 1어 22. 4.-19. 5. 2010, sinkhole 6; 5ㅇ, 1ð, 24. 3.-22. 4. 2010, 7우, 28. 6.-22. 7. 2010, sinkhole 7 .

Micrargus herbigradus (Blackwall, 1854)

Common epigeic species with Palearctic distribution, living in moss and damp leaves of deciduous and mixed forests.

Data: 1ㅇ, 24. 3.-22. 4. 2010, sinkhole 7.

Neriene clathrata (Sundevall, 1830)

Very abundant species in different forest habitats, often on wetlands, river banks, agrocenoses, meadows, and other unshaded habitats. 
Data: 1ô, 24. 3.-22. 4. 2010, sinkhole 2; 2ᄋ, 24. 3.22. 4. 2010, sinkhole 4; 3 , 24. 3.-22. 4. 2010, 1 , 19. 5.-28. 6. 2010, sinkhole 6.

Oedothorax agrestis (Blackwall, 1853)

Abundant species living in wet meadows, near swamps, ponds, and along streams.

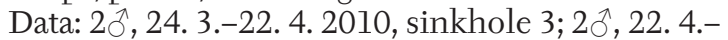
19. 5. 2010, sinkhole 6 .

\section{Oedothorax apicatus (Blackwall, 1850)}

Non-forest epigeic species that is dominant mainly in disturbed habitats of agrocenoses, cultural meadows, and at locations in the initial stage of succession. More abundant and more numerous than the aforementioned related species of Oedothorax agrestis.

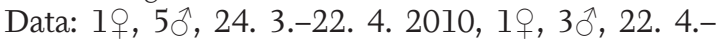
19. 5. 2010, 20, 19. 5.-28. 6. 2010, sinkhole 1; 9 ㅇ,

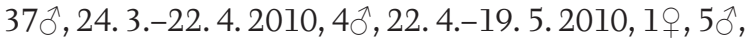

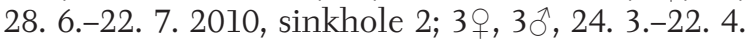

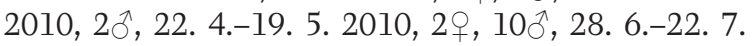
2010, sinkhole 3; 2 + , 5 $\hat{\text {, }, 24 . ~ 3 .-22 . ~ 4 . ~ 2010, ~ s i n k h o l e ~}$ 4; 6ㅇ, 8ภ, 24. 3.-22. 4. 2010, 30, 22. 4.-19. 5. 2010, $2 \hat{\jmath}$, 28. 6.-22. 7. 2010, sinkhole 5; 1우, 8, 24. 3.-22. 4.

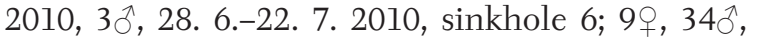
24. 3.-22. 4. 2010, 1우 9 ऊ , 22. 4.-19. 5. 2010, sinkhole 7.

\section{Pocadicnemis pumila (Blackwall, 1841)}

Abundant species of the forest and its surrounding, where it lives in the grass, moss, and debris. I It occurs sporadically also in open nonforest habitats.

Data: 1ㅇ, 26. 8.-22. 9.2010, sinkhole 5.

\section{Porrhomma errans (Blackwall, 1841), CR}

Very rare species with unclear environmental requirements. Firstly identified in the beet fields nearby to Olomouc (Miller 1974), later on rocky steppes of the Czech Central Mountains (Kůrka \& Buchar, 2010), and sandy marlite hillsides of eastern Bohemia (Dolanský, 2003). There are other recent data from Pálava in Moravia (Bryja et al., 2005) and especially from the Moravský kras PLA, where this species has been detected several times recently (Horáková, 2005; Niedobová et al., 2011), even in field habitats.

Data: 1ㅇ, 24. 3.-22. 4. 2010, sinkhole 3.

\section{Stemonyphantes lineatus (Linné, 1758)}

Moderately abundant species occurring in the grass undergrowth of xerothermic habitats, sporadically also on forest edges and sunlit meadow clearings.

Data: $8+$, 4ภ, 24. 3.-22. 4., 1ภ, 22. 4.-19. 5. 2010, sinkhole 2; 3 , 22. 4.-19. 5. 2010, sinkhole 3; 3ㅇ,

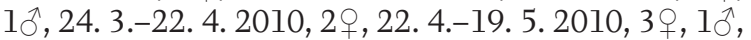
19. 5.-28. 6. 2010, sinkhole 4; 1, 1ठ, 24. 3.-22. 4. 2010, sinkhole 7.

Tapinocyba insecta (L. Koch, 1869)

Common Palaearctic species living in moss, leaves, and debris of forest and unshaded non-forest habitats. Except for western and southern Bohemia, it has a continuous distribution across most of Czech Republic.

Data: 2 , 22. 4.-19. 5. 2010, sinkhole 4.

Tenuiphantes cristatus (Menge, 1866)

Very abundant epigeic species, common in forest habitats.

Data: 1ð̂, 24. 3.-22. 4. 2010, sinkhole 7.

Walckenaeria monoceros (Wider, 1834), EN

Rare thermophilic species known only from a few localities in Czech thermophyticum (Valešová 1962; Potužáková 1975). It prefers well-preserved rocky steppes in lowland areas. There have not been any findings recorded from Moravia yet. A single adult male specimen was found in the outskirts of sinkhole No. 6.

Data: 1ㅇ, 24. 3.-22. 4. 2010, sinkhole 6.

\section{Tetragnathidae}

Pachygnatha clercki Sundevall, 1823

Abundant species living in wet leaves and debris of wet meadows, alder, and waterlogged forests.

Data: 1ㅇ, 24. 3.-22. 4. 2010, sinkhole 4; 2 , , 24. 3.22. 4. 2010; 19, 1ð , 22. 4.-19. 5. 2010, sinkhole 7.

Pachygnatha degeeri Sundevall, 1830

The most abundant species of the genus Pachygnatha living in detritus of various open habitats. Very abundant at mesophilic meadows, agrocenoses, clearings, and bright forest edges.

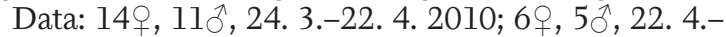

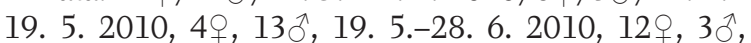
28. 6.-22. 7. 2010, 2ᄋ , 26. 8.-22. 9. 2010, sinkhole 1; 8 ㅇ, 5ð, 24. 3.-22. 4. 2010, 5ㅇ, 4ð, 22. 4.-19. 5. 2010, $3 \hat{\jmath}$, 28. 6.-22. 7. 2010, sinkhole 2; 6이 $11 \delta^{\lambda}$, 24. 3.22. 4. 2010, 2 ㅇ, 22. 4.-19. 5. 2010, 5ㅇ, 5 $\hat{\jmath}, 19.5 .-28.6$.

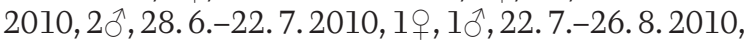

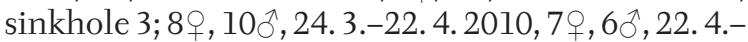

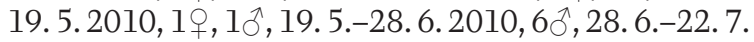
2010, 19, 20, 22. 7.-26. 8. 2010, sinkhole 4; 24우,

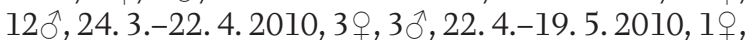

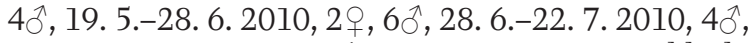
22. 7.-26. 8. 2010, 3ㅇ, 60े, 26. 8.-22.9. 2010, sinkhole

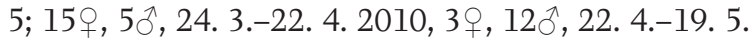

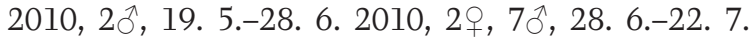

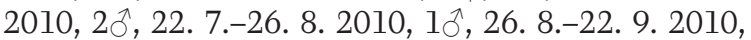

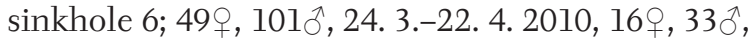

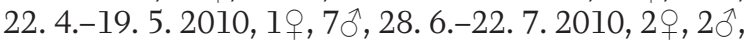
22. 7.-26. 8. 2010, sinkhole 7.

Pachygnatha listeri Sundevall, 1830

Scarce epigeic species living in moss and damp grass of deciduous and coniferous forests.

Data: 2 , 24. 3.-22. 4. 2010, sinkhole 1; 3 , 22. 4.19. 5. 2010, sinkhole 2; 1 이 $1 \hat{\jmath}$, 19. 5.-28. 6. 2010,

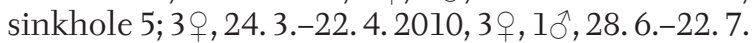
2010, sinkhole 5; 2 q , 3 3 , 24. 3.-22. 4. 2010, sinkhole 6; 3ㅇ, 24. 3.-22. 4. 2010, 19, 22. 4.-19. 5. 2010, sinkhole 7. 


\section{Lycosidae}

Alopecosa cuneata (Clerck, 1758)

Very common species in dry meadows and warm non-forested slopes, often in anthropogenically disturbed habitats. Data: 2 , , 24. 3-22. 4. 2010, 6o+ 22. 4.-19. 5. 2010, 1우 3수 19. 5.-28. 6. 2010, 1우, 26. 8.-22. 9. 2010, sinkhole 1; 6ㅇ, 22. 4.-19. 5. 2010, sinkhole $2 ; 2 \hat{\jmath}$, 24. 3.-22. 4. 2010, 3, 22. 4.-19. 5.

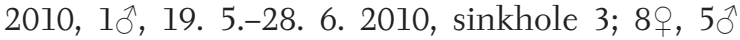
24. 3.-22. 4. 2010, 70ㅇ, 11 1 , 22. 4.-19. 5. 2010, 16우, 19. 5.-28. 6. 2010, 1어 $15 \hat{\jmath}$, 28. 6.-22. 7. 2010, 1 우, 2ิ, 22. 7.-26. 8. 2010, sinkhole 4; 34ㅇ, 3ð, 22. 4.-

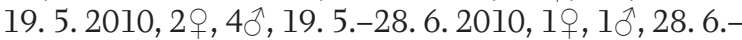
22. 7. 2010, sinkhole 5; 2 , 24. 3.-22. 4. 2010, 22 우,

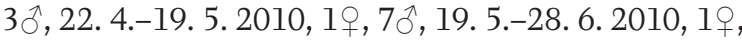
28. 6.-22. 7. 2010, sinkhole 6; 9, 24. 3.-22. 4. 2010, 43이 10, 22. 4.-19. 5. 2010, 19, 28. 6.-22. 7. 2010, sinkhole 7.

\section{Alopecosa pulverulenta (Clerck, 1758)}

Euryvalent species inhabiting many different habitats ranging from lowlands to mountains

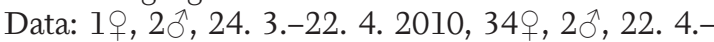
19. 5. 2010, 2 ㅇ, 5ㅅ, 19. 5.-28. 6. 2010, 2 우, 8, 28. 6.22. 7. 2010, 10 , 26. 8.-22. 9. 2010, sinkhole 1; 18우,

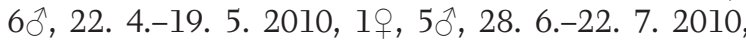

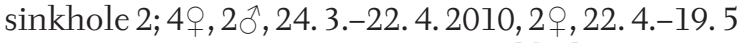
2010, 7ㅇ, 5^, 19. 5.-28. 6. 2010, sinkhole 3; 4q, 5ð, 24. 3.-22. 4. 2010, 8ㅇ, 10, 22. 4.-19. 5. 2010, 1우, 3스,

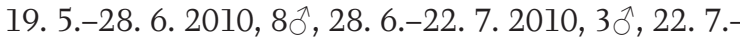
28. 6. 2010, sinkhole 4; 3 ㅇ, 24. 3.-22. 4. 2010, 3우, 8수,

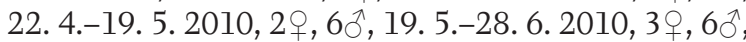
28. 6.-22. 7. 2010, 10, 22. 7.-26. 8. 2010, sinkhole 5; 1우 $2 \hat{\jmath}$, 24. 3.-22. 4. 2010, 8ㅇ, 22. 4.-19. 5. 2010, $2 \hat{\jmath}$, 19. 5.-28. 6. 2010, 1우 28. 6.-22. 7. 2010, sinkhole 6; 8ㅇ, 24. 3.-22. 4. 2010, 11우 22. 4.-19. 5. 2010, 90ิ, 28. 6.-22. 7.2010, sinkhole 7.

\section{Alopecosa trabalis (Clerck, 1758)}

Scarce species occurring in xerothermic habitats, in forests with southern exposure, and forest-steppe habitats in the warmer parts of the Czech Republic. It reached higher numbers only in the fourth and fifth sinkholes that were adjacent to forest habitat.

Data: 2 , 19. 5.-28. 6. 2010, 10, 22. 7.-28. 6. 2010, sinkhole 4; 3ㅇ, 24. 3.-22. 4. 2010, 1이 10, 22. 4.-19. 5 . 2010, 109, 2へ, 19. 5.-28. 6. 2010, 6へ, 28. 6.-22. 7. 2010, sinkhole 5; 1ㅇ, 24. 3.-22. 4. 2010, 1ठ, 22. 7.26. 8. 2010, sinkhole 6 .

\section{Aulonia albimana (Walckenaer, 1805)}

The smallest species of wolf spiders in our country, it lives in various open habitats from lowlands to mountains, more frequent occurrence at wet sites and slopes oriented to the north.

Data: 1우 28. 6.-22. 7. 2010, sinkhole 4; 2 , , 24. 3.22. 4. 2010, 19, 28. 6.-22. 7.2010, sinkhole 7 .

\section{Pardosa agrestis (Westring, 1861)}

Very abundant non-forest species in disturbed ruderal biotopes - ploughed areas, early stages of successional heaps, meadows, flooded meadows, etc. An interesting finding is mutual (less frequent) occurrence of closely related species Pardosa palustris, which was, however, much more numerous than P. agrestis.

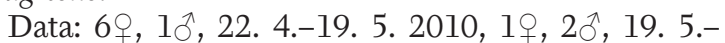
28. 6. 2010, 2 ㅇ, 4ð, 28. 6.-22. 7. 2010, sinkhole 1; 1우, 24. 3.-22. 4. 2010, 3․ 20. 22. 4.-19. 5. 2010, sinkhole 2; 3ㅇ, 19. 5.-28. 6. 2010, $2 \hat{\jmath}, 28$. 6.-22. 7. 2010,

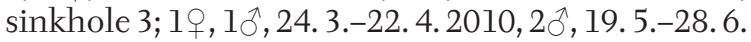
2010, sinkhole 4; 20 , 22. 7.-26. 8. 2010, sinkhole 5; 3 ㅇ, 24. 3.-22. 4. 2010, 1어, 20, 22. 4.-19. 5. 2010, 4우, 8ภ̂, 28. 6.-22. 7. 2010, sinkhole 6; 2 + , 22. 4.-19. 5. 2010, 5ㅇ, 6ふ, 28. 6.-22. 7. 2010, sinkhole 7.

\section{Pardosa amentata (Clerck, 1758)}

One of the most abundant spiders in the Czech Republic, it occupies a variety of wet habitats, such as river banks and water areas, wetlands or degraded peat bogs, where they move mostly just near the water surface. The second most common spider in the studied sinkholes, its most numerous representation was in the third sinkhole, which is formed by meadow vegetation with a strong representation of ruderal plants, especially with a majority of nitrophilous sorrel (Rumex spp.).

Data: 4 , $1{ }^{\lambda}$, 24. 3.-22. 4. 2010, $29+$, 22. 4.-19. 5.

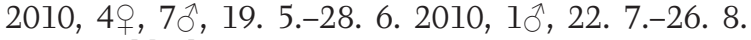

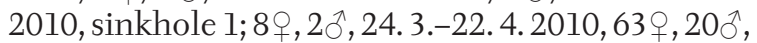
22. 4.-19. 5. 2010, 2ㅇ, 19. 5.-28. 6. 2010, 24우, 85 ऊ,, 28. 6.-22. 7. 2010, 3 3 , 22. 7.-26. 8. 2010, sinkhole 2; 17 , 14ð, 24. 3.-22. 4. 2010, 29 ㅇ, 22. 4.-19. 5. 2010,

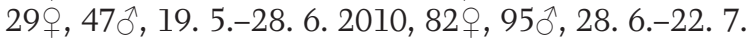
2010, $14 \circ, 42 \hat{\jmath}$, 22. 7.-26. 8. 2010, 2 ㅇ, 6ð, 26. 8.22. 9. 2010, sinkhole 3; 7ㅇ, 18 $\hat{\jmath}, 24$. 3.-22. 4. 2010,

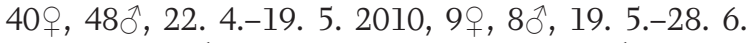
2010, 3ㅇ, 22へ, 28. 6.-22. 7. 2010, 19, 29ð, 22. 7.-

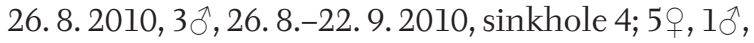
24. 3.-22. 4. 2010, 21 , 4 万人, 22. 4.-19. 5. 2010, 15 우, 5ภ, 19. 5.-28. 6. 2010, 10 ㅇ, 30ð, 28. 6.-22. 7. 2010, 6今, 22. 7.-26. 8. 2010, sinkhole 5; 10ㅇ, 10^, 24. 3.-

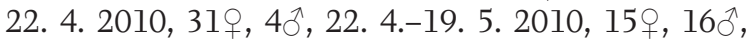

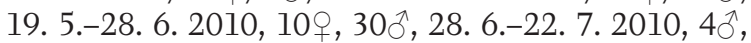
22. 7.-26. 8. 2010, 6\%, 26. 8.-22. 9. 2010, sinkhole 6; 18ㅇ, 7ðे, 22. 4.-19. 5. 2010, 4ㅇ, 28. 6.-22. 7. 2010, sinkhole 7 .

\section{Pardosa lugubris (Walckenaer, 1802)}

Very common species occurring in open bright sites, bright areas in forests, forest edges. It prefers wet sites.

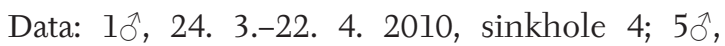
28. 6.-22. 7. 2010, sinkhole 5; 19, 28. 6.-22. 7. 2010, sinkhole 6 .

\section{Pardosa palustris (Linné, 1758)}

Abundant non-forest epigeic species typical for mesophilic meadows, common in agrocenoses and other disturbed habitats. The most abundant spider of the area, its high abundances formed a dominant component in each monitored sinkhole and almost a third of all the collected material of spiders.

Data: 3ㅇ, 4ภ, 24. 3.-22. 4. 2010, 26ㅇ, 4ภ, 22. 4.-

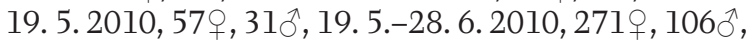

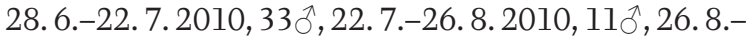




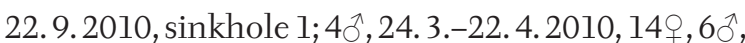

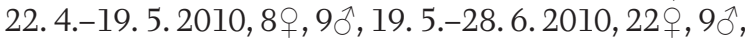
28. 6.-22. 7. 2010, 2 ऊ, 22. 7.-26. 8. 2010, sinkhole 2;

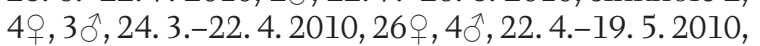
24ㅇ, 7ठ, 19. 5.-28. 6. 2010, 175ㅇ, 110今, 28. 6.-22. 7.

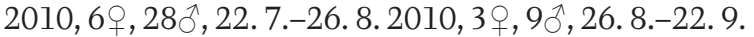

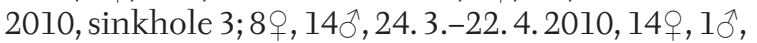
22. 4.-19. 5. 2010, 32우 9 9 , 19. 5.-28. 6. 2010, 44우,

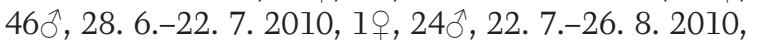
2 ㅇ, 2今, 26. 8.-22. 9. 2010, sinkhole 4; 4ㅇ, 24. 3.-22. 4.

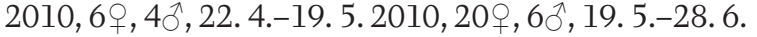
2010, 52ㅇ, 66 , 28. 6.-22. 7. 2010, 20, 22. 7.-26. 8. 2010, 1ㅇ, 2ð, 26. 8.-22. 9. 2010, sinkhole 5; 3ㅇ, 3ㅅ,

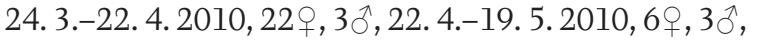
19. 5.-28. 6. 2010, 43ㅇ , 38 ठे, 28. 6.-22. 7. 2010, $15 \hat{\jmath}$, 22. 7.-26. 8. 2010, 1ㅇ, 26. 8.-22. 9. 2010, sinkhole 6;

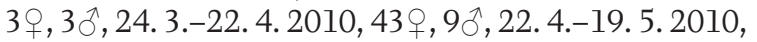
27우, 30今, 28. 6.-22. 7. 2010, 4우, $11 \jmath^{\lambda}, 22.7 .-26.8$. 2010, 1ठ, 26. 8.-22.9.2010, sinkhole 7.

\section{Pardosa pullata (Clerck, 1758)}

Very abundant species of a smallish wolf spiders living in both moist and dry habitats, forest edges from where they expand to agrocenoses too. Dominant occurrence mainly in flooded sinkholes with ruderal (nitrophilous) vegetation.

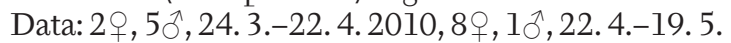

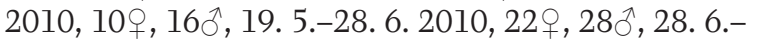
22. 7. 2010, 2 + $, 7 \hat{\jmath}, 22$. 7.-26. 8. 2010, 8ภ, 26. 8.22. 9. 2010, sinkhole 1; 50, 24. 3.-22. 4. 2010, 3 우,

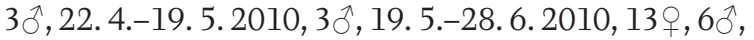
28. 6.-22. 7.2010, 4ㅇ, 5ð, 22. 7.-26. 8. 2010, sinkhole 2; 2 9 , 24. 3.-22. 4. 2010, 5ㅇ, 7ठ, 22. 4.-19. 5. 2010, 13 ㅇ, 5ภ, 19. 5.-28. 6. 2010, 22ㅇ, 30へ, 28. 6.-22. 7. 2010, 2ㅇ, 7ふे, 22. 7.-26. 8. 2010, sinkhole 3; 3ㅇ, $9 \hat{\jmath}$, 24. 3.-22. 4. 2010, 5ㅇ, 3 3 , 22. 4.-19. 5. 2010, 3 ㅇ, 6으, 19. 5.-28. 6. 2010, 11 ㅇ, 26수, 28. 6.-22. 7. 2010, 4우, $22 \widehat{\jmath}$, 22. 7.-26. 8. 2010, 2ᄋ, 20ิ, 26. 8.-22. 9. 2010, sinkhole 4; 2 ㅇ, 5ふ, 24. 3.-22. 4. 2010, 6ㅇ, 7ð, 22. 4.19. 5. 2010, 8 ㅇ, 6ᄎ, 19. 5.-28. 6. 2010, 11우 $26 \hat{\jmath}$,

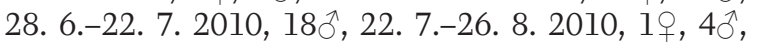
26. 8.-22. 9. 2010, sinkhole 5; 5ð, 24. 3.-22. 4. 2010,

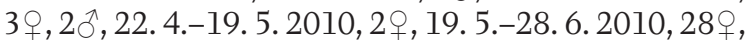
$18 \hat{\jmath}$, 28. 6.-22. 7. 2010, 4ㅇ, 19 0 , 22. 7.-26. 8. 2010, 2ิ, 26. 8.-22. 9. 2010, sinkhole 6; 2 \% , 24. 3.-22. 4.

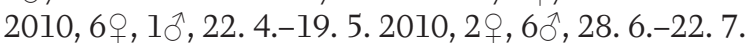

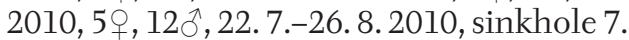

\section{Pardosa riparia (C. L. Koch, 1833)}

Scarce species on rocky steppes, forest clearings and edges of fields. It prefers preserved manundisturbed biotopes (Thaler \& Buchar, 1996). Detected only in sinkholes with a smaller proportion of nitrophilous vegetation.

Data: 1ô, 22. 7.-26. 8. 2010, sinkhole 4; 1ㅇ, 28. 6.22. 7. 2010, 3ㅇ, 22. 7.-26. 8. 2010, sinkhole 5; 2 우, 22. 7.-26. 8. 2010, sinkhole 6 .

\section{Trochosa ruricola (De Geer, 1778)}

Common and very abundant species living in grassy vegetation of wet meadows and fields. It prefers unshaded biotopes.
Data: 14ㅇ, 2ð, 24. 3.-22. 4. 2010, 3ㅇ, 19. 5.-28. 6.

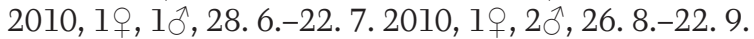

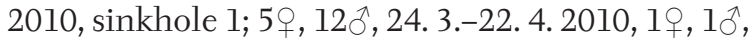
22. 4.-19. 5. 2010, 2 त, 28. 6.-22. 7. 2010, sinkhole 2;

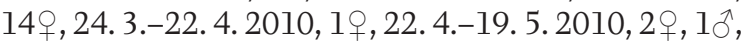
19. 5.-28. 6. 2010, 2 + , 28. 6.-22. 7. 2010, $2 \hat{\jmath}, 22$. 7.28. 6. 2010, sinkhole 3; 16ㅇ, 8ð, 24. 3.-22. 4. 2010,

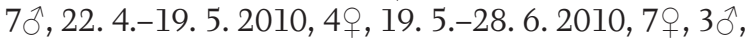
28. 6.-22. 7. 2010, 1ㅇ, 10ิ, 22. 7.-26. 8. 2010, sinkhole 4; 14우, 2ð, 24. 3.-22. 4. 2010, 4ㅇ, 1ð, 22. 4.-19. 5. 2010, 3ㅇ, 19. 5.-28. 6. 2010, 3ㅇ, 28. 6.-22. 7. 2010, 1ㅇ, $2 \jmath^{\lambda}, 22.7 .-26.8 .2010,2$ 이 $1 \jmath^{\lambda}, 26.8 .-22.9 .2010$, sinkhole 6; 8ㅇ, 3ð, 24. 3.-22. 4. 2010, 4ㅇ, 22. 4.-

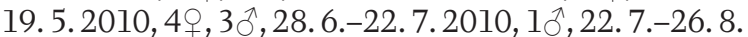
2010, sinkhole 7 .

\section{Trochosa terricola Thorell, 1856}

A very abundant species occurring in both dry and wet forests and their edges throughout the Czech Republic. It was numerous and dominant in all studied sinkholes.

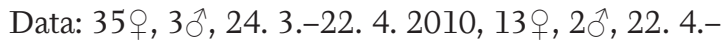
19. 5. 2010, 5 ㅇ, 5, 19. 5.-28. 6. 2010, 8ㅇ, 4^, 28. 6.-

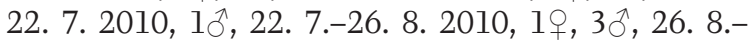
22. 9. 2010, sinkhole 1 ; 13 , 4 4 , 24. 3.-22. 4. 2010, 13우, 10, 22. 4.-19. 5. 2010, 1ㅇ, 19. 5.-28. 6. 2010, 9 ㅇ, 2犬, 28. 6.-22. 7. 2010, sinkhole 2; 12 ㅇ, $1 \hat{\jmath}, 24$. 3.-22. 4. 2010, 5ㅇ, 1ð, 22. 4.-19. 5. 2010, 5ㅇ, 5ð, 19. 5.-28. 6. 2010, 8ㅇ, 28. 6.-22. 7. 2010, 50, $2 \hat{0}$, 22. 7.26. 8. 2010, $20^{\hat{2}}$, 26. 8.-22. 9. 2010, sinkhole 3; 36우, 14ð, 24. 3.-22. 4. 2010, 17우, 2へ, 22. 4.-19. 5. 2010, 9 , 1ठ, 19. 5.-28. 6. 2010, 5ㅇ, 4へ, 28. 6.-22. 7. 2010,

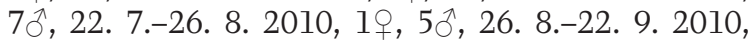
sinkhole 4; 34ㅇ, 4^, 24. 3.-22. 4. 2010, 13ㅇ, 4^, 22.

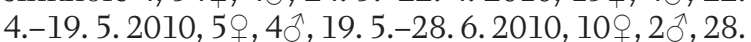

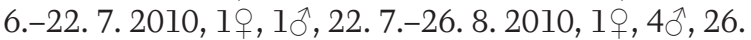
8.-22. 9. 2010, sinkhole 5; 13 q , 40, 24. 3.-22. 4. 2010,

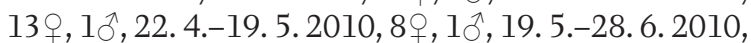

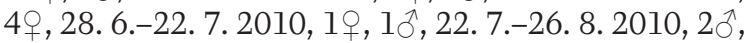
26. 8.-22. 9. 2010, sinkhole 6; 59, 20, 24. 3.-22. 4. 2010, 14ㅇ, 2へ, 22. 4.-19. 5. 2010, 2 ㅇ, 28. 6.-22. 7. 2010, 3ิे, 22. 7.-26. 8. 2010, 10ิ, 26. 8.-22. 9. 2010, sinkhole 7.

\section{Pisauridae}

Pisaura mirabilis (Clerck, 1758)

One of our most common spiders that inhabits open and moderately shaded habitats - forest edges, meadows, hillsides, light woods, rubble (mainly in nettles).

Data: 1ㅇ, 24. 3.-22. 4. 2010, 3 ㅇ, 19. 5.-28. 6. 2010,

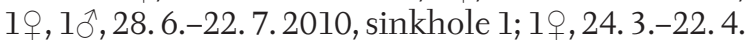
2010, sinkhole 2; 19, 24. 3.-22. 4. 2010, sinkhole 3; 2 , 24. 3.-22. 4. 2010, sinkhole 4; 5ㅇ, 24. 3.-22. 4. 2010, 2 ㅇ, 28. 6.-22. 7. 2010, sinkhole 5.

\section{Agelenidae}

Coelotes terrestris (Wider, 1834)

Very abundant species occurring in all types of forest habitats, especially in shaded locations.

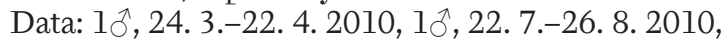
sinkhole 4; 2 \% , 26. 8.-22. 9. 2010, sinkhole 5. 
Malthonica campestris (C. L. Koch, 1834)

Scarce species living in shaded places in deciduous and coniferous forests. Only one specimen found in the sinkhole on meadow habitat adjacent to a deciduous forest.

Data: 1ठึ, 28. 6.-22. 7. 2010, sinkhole 5.

\section{Dictynidae}

Cicurina cicur (Fabricius, 1793)

Abundant species living in grass and moss of shady deciduous and coniferous forests. Species characterized by its autumn activity, the adults mature in winter (Tretzel, 1954).

Data: 1ô, 24. 3.-22. 4. 2010, 1ㅇ, 22. 4.-19. 5. 2010, sinkhole 2; 30, 26. 8.-22. 9. 2010, sinkhole 3; 1 으, 22. 7.-26. 8. 2010, sinkhole 4; 1 त, 22. 7.-26. 8. 2010,

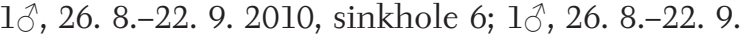
2010, sinkhole 7 .

\section{Amaurobiidae}

Callobius claustrarius (Hahn, 1833)

Moderately abundant species living under stones and bark of trees in preserved forest habitats. Three specimens only were detected in a sinkhole near forest.

Data: 1ㅇ, 24. 3.-22. 4. 2010, 2ð, 22. 7.-26. 8. 2010, sinkhole 4.

\section{Liocranidae}

Agroeca brunnea (Blackwall, 1833)

Very abundant species equally distributed throughout the Czech Republic. It is abundant especially in deciduous and coniferous forests, waterlogged meadows, and peat bogs.

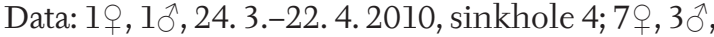
24. 3.-22. 4. 2010, sinkhole 5; 1ㅇ, 24. 3.-22. 4. 2010, sinkhole 6; 2 + , 24. 3.-22. 4. 2010, sinkhole 7 .

\section{Gnaphosidae}

Drassodes lapidosus (Walckenaer, 1802)

The most abundant representative of the Gnaphosidae family that occurs in most xerothermal habitats. One adult female specimen only detected in the peripheral part of the first sinkhole.

Data: 1ố, 28. 6.-22. 7.2010, sinkhole 1.

Drassyllus praeficus (L. Koch, 1866)

Abundant species of open habitats, very common in grassland habitats, forest clearings and edges.

Data: 1ô, 22. 7.-26. 8. 2010, sinkhole 5.

Drassyllus pusillus (C. L. Koch, 1833)

Abundant euryvalent species occurring in both xerothermic and waterlogged habitats, forest edges, dumps, agrocenoses, and other ruderal sites.

Data: 1ㅇ, 28. 6.-22. 7. 2010, sinkhole 7.

Haplodrassus signifer (C. L. Koch, 1839)

Very abundant epigeic species common in various open as well as forest habitats.

Data: 2 , 28. 6.-22. 7. 2010, sinkhole $1 ; 3 \circ, 2 \hat{\jmath}$, 22. 4.-19. 5. 2010, sinkhole 5; 1, 24. 3.-22. 4. 2010, sinkhole 6; 1ㅇ, 24. 3.-22. 4. 2010, sinkhole 7 .
Zelotes latreillei (Simon, 1878)

Abundant epigeic species preferring open habitats.

Data: 2ㅇ, 24. 3.-22. 4. 2010, sinkhole 1; 4ㅇ, 24. 3.22. 4. 2010, 1 ㅇ 19. 5.-28. 6. 2010, sinkhole 4; 2 의, 24. 3.-22. 4. 2010, sinkhole 5; 19, 24. 3.-22. 4. 2010, 2 ㅇ, 1ð̂, 22. 4.-19. 5. 2010, sinkhole 6; 1ㅇ, 24. 3.-22. 4. 2010, sinkhole 7.

\section{Zelotes longipes (L. Koch, 1866)}

Rare relict species recorded in preserved xerothermic sites of the Czech and Moravian thermophyticum. Typically autumn species (Noflatcher 1988).

Data: 1 ㅇ, 24. 3.-22. 4. 2010, sinkhole 2; 2 , 22. 4.19. 5. 2010, sinkhole 4 .

\section{Zoridae}

Zora spinimana (Sundevall, 1833)

Abundant species living in debris and leaves of damp shady woods, in open habitats occurs in wet meadows, marshes and peat bogs.

Data: 19, 24. 3.-22. 4. 2010, 10, 22. 4.-19. 5. 2010, 2 ㅇ, 19. 5.-28. 6. 2010, 2 ㅇ, 28. 6.-22. 7. 2010, sinkhole 1; 2 + $, 1 \jmath^{\top}, 24.3 .-22.4$. 2010, sinkhole 2.

\section{Philodromidae}

Philodromus collinus C. L. Koch, 1835

Very abundant species living in vegetation in forests (most numerous in spruce forests) as well as in various open habitats.

Data: 1ठ, 28. 6.-22. 7.2010, sinkhole 5.

Thanatus formicinus (Clerck, 1758)

Abundant species characteristic for ground level of rock steppes, heathland, and forest steppes.

Data: 1o, 24. 3.-22. 4. 2010, sinkhole 3; 3우, 24. 3.-22. 4. 2010, sinkhole 4; 19, 24. 3.-22. 4. 2010, sinkhole 5; 3 ㅇ, 10, 28. 6.-22. 7. 2010, sinkhole 6 .

\section{Thomisidae}

Ozyptila atomaria (Panzer, 1801)

Rare species living among detritus on rock steppes and other xerothermic habitats.

Data: 1ㅇ, 24. 3.-22. 4. 2010, sinkhole 4; 2 , 24. 3.22. 4. 2010, sinkhole 5 .

Ozyptila trux (Blackwall, 1846)

Very abundant species living among detritus and litter on marshy pond margins and in wet meadows.

Data: 1ô, 26. 8.-22. 9. 2010, sinkhole 6.

Xysticus audax (Schrank, 1803)

Very abundant and common species living in low vegetation in various open and forest habitats.

Data: 5ㅇ, 22. 4.-19. 5. 2010, 3 \% , 28. 6.-22. 7. 2010, 1ठ, 22. 7.-26. 8. 2010, sinkhole 1; 19, 22. 4.-19. 5. 2010, 2ิ, 22. 7.-26. 8. 2010, sinkhole 3; 1ㅇ, 19. 5.28. 6. 2010, 1ㅇ 28. 6.-22. 7. 2010, sinkhole 4; 4우, 22. 4.-19. 5. 2010, sinkhole 7.

Xysticus bifasciatus C. L. Koch, 1837

Very abundant and very common species living in grass in meadows and other open habitats. Bryja 
et al. (2005) reported this species as rare in the area of southern Moravia.

Data: 2へ, 24. 3.-22. 4. 2010, 4, 1ठ, 19. 5.-28. 6. 2010, 3ㅇ, 1§, 28. 6.-22. 7. 2010, sinkhole 1; 2 ㅇ, 22. 4.-19. 5. 2010, sinkhole 2 ; $1 \hat{\jmath}, 22.4 .-19.5 .2010$, 1 , , 22. 7.-26. 8. 2010, sinkhole 3; 3ㅇ, 1ð, 24. 3.-22. 4. 2010, 5ㅇ, 2^, 22. 4.-19. 5.2010,3ㅇ, 19. 5.-28.6.2010, sinkhole 4; 13ㅇ, 3ત, 22. 4.-19. 5. 2010, 10ㅇ, 19. 5.28. 6. 2010, 4우 $2{ }^{\jmath}$, 28. 6.-22. 7. 2010, sinkhole 5; 7 우, 19. 5-22. 7. 2010, 1ㅇ, 26. 8.-22. 9. 2010, sinkhole 6;

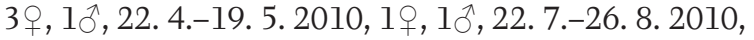
sinkhole 7.

\section{Xysticus cristatus (Clerck, 1758)}

Very abundant and common species living in grass in meadows and other open habitats as well as in fields and in orchards.

Data: 2 ㅇ, 24. 3.-22. 4. 2010, 7ㅇ, 22. 4.-19. 5. 2010,

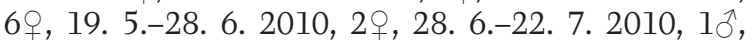
22. 7.-26. 8. 2010, sinkhole 1; 1우 28. 6.-22. 7. 2010, 1 ㅇ, 22. 7.-26. 8. 2010, sinkhole $2 ; 3$, $1{ }^{\lambda}, 22.4 .-19.5$. 2010, 3ㅇ, 19. 5.-28. 6. 2010, 19, 28. 6.-22. 7. 2010, 1우, 22. 7.-26. 8. 2010, sinkhole 3; 2 어, 1ㅅ, 24. 3.-22. 4. 2010, 11ㅇ, 22. 4.-19. 5. 2010, sinkhole 4; 2 , , 24. 3.-

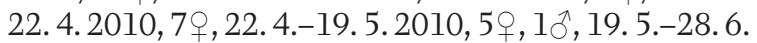
2010, 2 + , 28. 6.-22. 7. 2010, sinkhole 5; 3ㅇ, 24. 3.22. 4. 2010, 2 ㅇ, 22. 4.-19. 5. 2010, 1ㅇ, $2 \hat{0}, 19.5 .-28.6$. 2010, 1ㅇ, 28. 6.-22. 7. 2010, sinkhole 6; 5ㅇ, 24. 3.22. 4. 2010, 7우 22. 4.-19. 5. 2010, 1ㅇ, 22. 7.-26. 8. 2010, sinkhole 7 .

Xysticus kochi Thorell, 1872

Abundant, in grass and on vegetation on rock steppes, meadows, in urban grasslands, in orchards and gardens.

Data: 1ㅇ, 24. 3.-22. 4. 2010, 6ㅇ, 22. 4.-19. 5. 2010,

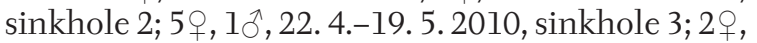
24. 3.-22. 4. 2010, 19 , 22. 4.-19. 5. 2010, sinkhole 4; 4ㅇ, 22. 4.-19. 5. 2010, sinkhole 5; 1이 19. 5.-28. 6. 2010, sinkhole 6 .

\section{Salticidae}

Evarcha arcuata (Clerck, 1758)

Very abundant species living in herb vegetation in both wet and xerothermic meadows and in other open habitats.

Data: 1ㅇ, 26. 8.-22. 9. 2010, sinkhole 5.

Phlegra fasciata (Hahn, 1826)

Abundant and sometimes common species living in grass on rock steppes and xerothermic slopes, in forest steppes and forest edges, also on spoil heaps and road verges.

Data: 2 , , 22. 7.-26. 8. 2010, sinkhole 4.

\section{DISCUSSION}

The study found no significant number of species but, generally, species of cultural landscapes. Several findings are interesting, e.g. the discovery of Porrhomma erans. This species is very rare in the
Czech Republic (Buchar \& Růžička, 2002), but it has also been reported previously in the monitored area (Horáková 2005; Niedobová at al., 2011). In the Czech Republic, this species was first recorded by Miller (1974) from beet fields, and other published findings of this species come from fields as well (Roberts 1987, 1995; Řezáč et al., 2006; Öberg et al., 2008; British Arachnological Society, 2011). We believe that it is unreasonable to include this species in the Red List of invertebrates of the Czech Republic (Růžička, 2005) at all, it is very rare species although common habitats like arable fields and it is impossible to manage or protect habitats according to its presence.

Walckenaeria monoceros is another species important from the faunistic perspective. This species is more commonly observed in xerothermic habitats (Buchar \& Růžička, 2002), such as rocky steppes, but only from the so called Czech thermophyticum (sensu Slavik, 1984). This is the first finding of $W$. monoceros in the area of Moravia. To date, all findings of this thermophilous species have been recorded from the western part of Czech thermophyticum. Zelotes longipes is among the other species important from the faunistic perspective. From the Moravský kras PLA, it was reported only from Hádecká planinka (Kůrka, 1994). Recently, it has also been reported from the hillside of Macošská stráň at Vilémovice (Niedobová et al., 2011). The same situation is in case of record of Mecopisthes silus. However, Linyphiidae family representatives very often migrate aeronautically by wind, which can result in spreading and settling in the nearby as well as more distant areas.

The other species are very abundant and most of them are typical for agrarian landscape. However, typically steppe and forest-steppe species (e.g. Alopecosa trabalis or Pardosa riparia) and forest species (Coelotes terrestris, Pardosa lugubris, Agroeca brunnea, and Callobius claustrarius) were also recorded. All these species were found near the forest $(100 \mathrm{~m}$ from the edge). Their presence indicates that in the case of appropriately adjusted conditions and management in sinkholes, these can be populated by both forest and steppe species. On the other hand, a typical meadow species Pachygnatha degeeri does occur in sinkholes in a limited number, but it is much more abundant mainly in and around their edges. At the same time, very abundant wolf spiders Alopecosa cuneata, A. pulverulenta, and Pardosa amentata occur mainly in sinkholes without any significant nitrophilous vegetation cover, such as Rumex spp. or the Urtica dioica nettle.

We can conclude that there is a relatively valuable fauna in the sinkholes and that there are suitable conditions for rare species which cannot survive in an open farmland. On the other hand, sinkholes don't increase total number of species in open agricultural areas of Moravský kras PLA. 
I: Summary of species recorded - Relictness (Buchar 1983): RI (relict species of type 1), RII (relict species of type 2), E (euryvalent species); Habitat preferences (Buchar \& Růzička, 2002): C (climax), SN (seminatural), D (disturbed); Thermopreferences (Buchar\& Rüzička, 2002): $T$ - thermo, $M$ - meso, $O$ - oreo. Bold means main preference, brackets mean minority preference.

\begin{tabular}{|c|c|c|c|c|c|}
\hline Species & q & $\hat{\sigma}$ & Relictness & $\begin{array}{c}\text { Habitat } \\
\text { preferences }\end{array}$ & Thermopreferences \\
\hline \multicolumn{6}{|l|}{ Mimetidae } \\
\hline Ero furcata (Villers, 1789) & 0 & 1 & RII & $\mathrm{C}, \mathrm{SN}$ & $\mathrm{T}, \mathbf{M}$ \\
\hline \multicolumn{6}{|l|}{ Linyphiidae } \\
\hline Bathyphantes nigritus (Westring, 1851) & 1 & 0 & RII & $\mathrm{C}, \mathrm{SN}$ & $\mathrm{T}, \mathbf{M}, \mathrm{O}$ \\
\hline Centromerita bicolor (Blackwall, 1833) & 3 & 0 & $\mathrm{E}$ & C, SN, D & M, O \\
\hline Centromerus sylvaticus (Blackwall, 1841) & 13 & 3 & $\mathrm{E}$ & C, SN, D & $\mathrm{T}, \mathrm{M}, \mathrm{O}$ \\
\hline Diplocephalus cristatus (Blackwall, 1833) & 5 & 0 & $\mathrm{E}$ & C, SN, D & M, O \\
\hline Diplocephalus picinus (Blackwall, 1841) & 20 & 1 & RII & $\mathrm{C}, \mathrm{SN}$ & $\mathrm{T}, \mathrm{M}, \mathrm{O}$ \\
\hline Diplostyla concolor (Wider, 1834) & 9 & 3 & $\mathrm{E}$ & $\mathrm{C}, \mathrm{SN}$ & $\mathrm{T}, \mathrm{M}, \mathrm{O}$ \\
\hline Erigone atra Blackwall, 1833 & 111 & 10 & $\mathrm{E}$ & C, SN, D & $\mathrm{T}, \mathrm{M}, \mathrm{O}$ \\
\hline Maso sundevalli (Westring, 1851) & 4 & 2 & RII & $\mathrm{C}, \mathrm{SN}$ & $\mathrm{T}, \mathrm{M}, \mathrm{O}$ \\
\hline Mecopisthes silus (O. P.-Cambridge, 1872) & 1 & 0 & RI & $\mathrm{C}$ & M \\
\hline Meioneta rurestris (C. L. Koch, 1836) & 14 & 1 & $\mathrm{E}$ & C, SN, D & $\mathrm{T}, \mathrm{M}, \mathrm{O}$ \\
\hline Micrargus herbigradus (Blackwall, 1854) & 1 & 0 & $\mathrm{E}$ & C, SN, D & $\mathrm{T}, \mathrm{M}, \mathrm{O}$ \\
\hline Neriene clathrata (Sundevall, 1830) & 6 & 1 & RII & $\mathrm{C}, \mathrm{SN}$ & $\mathrm{T}, \mathbf{M}, \mathrm{O}$ \\
\hline Oedothorax agrestis (Blackwall, 1853) & 0 & 4 & RII & $\mathrm{C}, \mathrm{SN}$ & $\mathbf{M}, \mathrm{O}$ \\
\hline Oedothorax apicatus (Blackwall, 1850) & 36 & 143 & E & C, SN, D & $\mathrm{T}, \mathrm{M}$ \\
\hline Pocadicnemis pumila (Blackwall, 1841) & 1 & 0 & $\mathrm{E}$ & $\mathrm{C}, \mathrm{SN}$ & $\mathrm{T}, \mathbf{M}$ \\
\hline Porrhomma errans (Blackwall, 1841) & 1 & 0 & $\mathrm{E}$ & C, D & $\mathrm{T}$ \\
\hline Stemonyphante lineatus (Linné, 1758) & 20 & 8 & $\mathrm{E}$ & C, SN, D & $\mathrm{T}, \mathbf{M}$ \\
\hline Tapinocyba insecta (L. Koch, 1869) & 0 & 2 & RII & C, SN & $\mathrm{T}, \mathbf{M}$ \\
\hline Tenuiphantes cristatus (Menge, 1866) & 0 & 1 & RII & $\mathrm{C}, \mathrm{SN}$ & M, O \\
\hline Walckenaeria monoceros (Wider, 1834) & 1 & 0 & RI & $\mathrm{C}$ & $\mathrm{T}$ \\
\hline \multicolumn{6}{|l|}{ Tetragnathidae } \\
\hline Pachygnatha clercki Sundevall, 1823 & 4 & 1 & $\mathrm{E}$ & C, SN, D & $\mathrm{T}, \mathbf{M}$ \\
\hline Pachygnatha degeeri Sundevall, 1830 & 203 & 295 & $\mathrm{E}$ & C, SN, D & $\mathrm{T}, \mathbf{M}$ \\
\hline Pachygnatha listeri Sundevall, 1830 & 18 & 5 & RII & $\mathrm{C}, \mathrm{SN}$ & $\mathrm{T}, \mathbf{M}$ \\
\hline \multicolumn{6}{|l|}{ Lycosidae } \\
\hline Alopecosa cuneata (Clerck, 1758) & 231 & 58 & E & $\mathrm{C}, \mathrm{SN}$ & $\mathrm{T}, \mathrm{M}, \mathrm{O}$ \\
\hline Alopecosa pulverulenta (Clerck, 1758) & 124 & 90 & $\mathrm{E}$ & C, SN, D & $\mathrm{T}, \mathrm{M}, \mathrm{O}$ \\
\hline Alopecosa trabalis (Clerck, 1758) & 18 & 10 & RII & $\mathrm{C}, \mathrm{SN}$ & $\mathrm{T}, \mathrm{M}$ \\
\hline Aulonia albimana (Walckenaer, 1805) & 4 & 0 & RII & $\mathrm{C}, \mathrm{SN}$ & $\mathrm{T}, \mathbf{M}$ \\
\hline Pardosa agrestis (Westring, 1861) & 34 & 30 & $\mathrm{E}$ & SN, D & $\mathrm{T}, \mathbf{M}$ \\
\hline Pardosa amentata (Clerck, 1758) & 506 & 574 & $\mathrm{E}$ & C, SN, D & $\mathrm{T}, \mathrm{M}, \mathrm{O}$ \\
\hline Pardosa lugubris (Walckenaer, 1802) & 1 & 6 & RII & C, SN, D & $\mathrm{T}, \mathrm{M}, \mathrm{O}$ \\
\hline Pardosa palustris (Linné, 1758) & 975 & 672 & E & C, SN, D & $\mathrm{T}, \mathrm{M}, \mathrm{O}$ \\
\hline Pardosa pullata (Clerck, 1758) & 214 & 335 & $\mathrm{E}$ & C, SN, D & $\mathrm{T}, \mathrm{M}, \mathrm{O}$ \\
\hline Pardosa riparia (C. L. Koch, 1833) & 6 & 1 & RI & $\mathrm{C}, \mathrm{SN}$ & $\mathrm{T}, \mathrm{M}, \mathrm{O}$ \\
\hline Trochosa ruricola (De Geer, 1778) & 115 & 55 & $\mathrm{E}$ & C, SN, D & $\mathrm{T}, \mathrm{M}, \mathrm{O}$ \\
\hline Trochosa terricola Thorell, 1856 & 325 & 105 & E & C, SN, D & $\mathrm{T}, \mathrm{M}$ \\
\hline \multicolumn{6}{|l|}{ Pisauridae } \\
\hline Pisaura mirabilis (Clerck, 1758) & 16 & 1 & RII & C,SN,D & $\mathrm{T}, \mathbf{M}$ \\
\hline \multicolumn{6}{|l|}{ Agelenidae } \\
\hline Coelotes terrestris (Wider, 1834) & 2 & 2 & RII & $\mathrm{C}, \mathrm{SN}$ & $\mathrm{T}, \mathrm{M}, \mathrm{O}$ \\
\hline Malthonica campestris (C.L. Koch, 1834) & 0 & 1 & RII & $\mathrm{C}, \mathrm{SN}$ & $\mathrm{T}, \mathrm{M}$ \\
\hline
\end{tabular}




\begin{tabular}{|c|c|c|c|c|c|}
\hline Species & 우 & $\hat{\sigma}$ & Relictness & $\begin{array}{c}\text { Habitat } \\
\text { preferences }\end{array}$ & Thermopreferences \\
\hline \multicolumn{6}{|l|}{ Dictynidae } \\
\hline Cicurina cicur (Fabricius, 1793) & 2 & 7 & $\mathrm{E}$ & C, SN, D & $\mathrm{T}, \mathbf{M}$ \\
\hline \multicolumn{6}{|l|}{ Amaurobiidae } \\
\hline Callobius claustrarius (Hahn, 1833) & 1 & 2 & RII & $\mathrm{C}, \mathrm{SN}$ & M, O \\
\hline \multicolumn{6}{|l|}{ Liocranidae } \\
\hline Agroeca brunnea (Blackwall, 1833) & 11 & 4 & RII & $\mathrm{C}, \mathrm{SN}$ & $\mathrm{T}, \mathbf{M}$ \\
\hline \multicolumn{6}{|l|}{ Gnaphosidae } \\
\hline Drassodes lapidosus (Walckenaer, 1802) & 0 & 1 & RII & $\mathrm{C}, \mathrm{SN}$ & $\mathrm{T}, \mathrm{M}$ \\
\hline Drassyllus praeficus (L. Koch, 1866) & 0 & 1 & RII & C, SN & $\mathrm{T}, \mathbf{M}$ \\
\hline Drassyllus pusillus (C. L. Koch, 1833) & 1 & 0 & $\mathrm{E}$ & C, SN, D & $\mathrm{T}, \mathrm{M}$ \\
\hline Haplodrassus signifer (C. L. Koch, 1839) & 7 & 2 & $\mathrm{E}$ & C, SN, D & $\mathrm{T}, \mathrm{M}, \mathrm{O}$ \\
\hline Zelotes latreillei (Simon, 1878) & 13 & 1 & RII & C, SN, D & $\mathrm{T}, \mathbf{M}$ \\
\hline Zelotes longipes (L. Koch, 1866) & 3 & 0 & RI & $\mathrm{C}$ & $\mathrm{T}, \mathrm{M}$ \\
\hline \multicolumn{6}{|l|}{ Zoridae } \\
\hline Zora spinimana (Sundevall, 1833) & 7 & 2 & RII & C, SN, D & $\mathrm{T}, \mathbf{M}, \mathrm{O}$ \\
\hline \multicolumn{6}{|l|}{ Philodromidae } \\
\hline Philodromus collinus C. L. Koch, 1835 & 0 & 1 & RII & $\mathrm{C}, \mathrm{SN}$ & $\mathrm{T}, \mathbf{M}, \mathrm{O}$ \\
\hline Thanatus formicinus (Clerck, 1758) & 8 & 1 & E & $\mathrm{C}, \mathrm{SN}$ & $\mathrm{T}, \mathrm{M}$ \\
\hline \multicolumn{6}{|l|}{ Thomisidae } \\
\hline Ozyptila atomaria (Panzer, 1801) & 3 & 0 & RII & $\mathrm{C}, \mathrm{SN}$ & $\mathrm{T}, \mathrm{M}$ \\
\hline Ozyptila trux (Blackwall, 1846) & 0 & 1 & $\mathrm{E}$ & C, SN, D & M, O \\
\hline Xysticus audax (Schrank, 1803) & 15 & 3 & $\mathrm{E}$ & $\mathrm{C}, \mathrm{SN}$ & $\mathrm{T}, \mathbf{M}, \mathrm{O}$ \\
\hline Xysticus bifasciatus C. L. Koch, 1837 & 60 & 15 & E & C, SN, D & $\mathrm{T}, \mathbf{M}, \mathrm{O}$ \\
\hline Xysticus cristatus (Clerck, 1758) & 76 & 6 & $\mathrm{E}$ & C, SN, D & $\mathrm{T}, \mathbf{M}, \mathrm{O}$ \\
\hline Xysticus kochi Thorell, 1872 & 20 & 1 & $\mathrm{E}$ & C, SN, D & $\mathrm{T}, \mathrm{M}$ \\
\hline \multicolumn{6}{|l|}{ Salticidae } \\
\hline Evarcha arcuata (Clerck, 1758) & 1 & 0 & RII & C, SN & $\mathrm{T}, \mathbf{M}$ \\
\hline Phlegra fasciata (Hahn, 1826) & 2 & 0 & $\mathrm{E}$ & $\mathrm{C}, \mathrm{SN}$ & $\mathrm{T}, \mathrm{M}$ \\
\hline
\end{tabular}

\section{SUMMARY}

During the arachnological research of sinkhole area from 24 March 2010 to 22 September 2010, a total number of 5742 adult spiders were collected. Out of this number, 59 species belonging to 14 families were determined. In addition, 687 juvenile specimens were also found. The crucial records are as follows: Porrhomma errans - very rare species known from different habitats (beet fields, steppe habitats in České stř̌edohoří PLA, Moravský kras PLA, and Pálava PLA, and meadows in Moravský kras PLA); Walckenaeria monoceros - from the Czech Republic, species known mainly from Bohemia, where prefers xerothermic habitats in Bohemian termophyticum.

According to thermopreference assesment, the highest number of species is mesophilic (55\%) and thermophilic (30\%), the remaining (15\%) species are psychophilic. In the area of Moravský kras PLA, thermophilnous species can be found mainly in the southern part, towards the massif of Hády. To the north of the studied sinkholes, the biotope gradually changes into mesophyticum, which is evident in more than half representation of mesophilic species. Solely thermophilic species are represented by Porrhomma errans and Walckenaeria monoceros which are among the most important findings in the surveyed area There are suitable conditions in the sinkholes, enabling a survive of relatively valuable fauna. On the other hand, sinkholes cannot increase the biodiversity in an agrarian landscape because of their relatively small size.

\section{Acknowledgment}

This study was supported by project IGA MENDELU No. TP05/2011. We thank especially to Jana Niedobová, Jan Bezděk, Lukáš Nytra, Jiř́i Mrtka, Martin Řezníček and Romana Kunderová for their help in the field and laboratory. Last but not least thanks belong to Petr Dolejš (National Museum, Prague) for his valuable comments. 


\section{REFERENCES}

BRYJA, V., SVATOŇ, J., CHYTIL, J., MAJKUS, Z., RŮŽIČKA, V., KASAL, P., DOLANSKÝ, J., BUCHAR, J., CHVÁTALOVÁ, I., ŘEZÁČ, M., KUBCOVÁ, L., ERHART, J., FENCLOVÁ, I., 2005: Spiders (Araneae) of the Lower Morava Biosphere Reserve and closely adjacent localities (Czech republic). Acta Musei Moraviae, Scientiae biologicae (Brno), 90: 13-184.

BRITISH ARACHNOLOGICAL SOCIETY, 2011: Spider and Harvestman Recording Scheme website - the national recording schemes for spiders and harvestmen in Britain. Available online at: http://srs.britishspiders.org.uk/portal. php/p/Welcome (cited: 21. 7. 2011).

BUCHAR, J., 1983: Klasifikace druhů pavoučí zvířeny Čech, jako pomůcka k bioindikaci kvality životního prostředí. [Die Klassifikation der Spinnenarten Böhmens als ein Hilfsmittel für die Bioindikation der Umwelt]. Fauna Bohemiae Septentrionalis, 8: 119-135.

BUCHAR, J., RŮŽICKA, V., 2002: Catalogue of Spiders of the Czech Republic. Peres, Praha, $351 \mathrm{pp}$.

DOLANSKÝ, J., 2003: Arachnofauna písčin a bílých strání východních Čech. (Spiders of Eastbohemian sandy habitats and sliding chalk slopes). Východočeský sborník prírodovédecký - Práce a studie, 10: 285-310.

HORÁKOVÁ, J., 2005: Vybrané skupiny epigeické fauny závrtů CHKO Moravský kras a jejich antropogenní ovlivnění. [Selected groups of epigeal invertebrate fauna of sink hole in Moravský kras PLA and their anthropogenetic involvement]. Ph.D. thesis, AF MZLU Brno, 248 pp.

HORÁKOVÁ, J., HULA, V., PIKULA, J., 2005: Contribution to fauna of invertebrates of sink holes within the agricultural landscape of the Moravian KarstProtected Area. Part one: Carabidae (Coleoptera). Acta univ. agric. et silvic. Mendel. Brun. (Brno), 52(5): 53-62.

ICZN, 1999: International Code of Zoological Nomenclature. Fourth Edition. International Trust for Zoological Nomenclature, London, 306 pp.

KŮRKA, A., 1982: Pavouci (Araneida) vrchu Stožec na Šumavě. (Spinnen (Araneida) auf dem Stožec im Böhmerwald). Acta Musei Naturae Pragae, 38: 4778.

KU゚RKA, A., 1994: Přehled druhů pavouků (Araneida) ve sbírce prof. F. Millera (zoologické sbírky Přírodovědeckého muzea - Národního muzea), část I. (A survey of spider species (Araneida) in Prof. F. Miller's collection (Department of Zoology, Museum of Natural History - National Musem), Part I). Časopis Národního muzea, Řada přirodovédná, 163: 43-54.

KU゚RKA, A., BUCHAR, J., 2010: Pavouci (Araneae) vrchu Oblík v Českém středohoří (severozápadní Čechy). Spiders (Araneae) of the Oblík Hill in the České středohoří Highlands (Northwestern
Bohemia, Czech Republic). Acta Musei Bohemiae Borealis, Scientiae Naturales, 28: 71-106.

MILLER, F., 1951: Pavoučí zvířena vrchoviště u Rejvízu v Jesenících. (Spider fauna of the PeatBogs near Rejvíz). Př́rodovédný sborník Ostravského kraje, 12: 202-247.

MILLER, F., 1974: Pavoučí fauna řepných polí v okolí Chválkovic a Nákla na Hané. (Spider fauna of sugar beet fields in the surroundings of Chválkovice and Náklo in Haná). Acta Univerzity Palackého Olomouc, Faculta rerum naturae Biologica, 15: 175-181.

NIEDOBOVÁ, J., HULA, V., ŠŤASTNÁ, P., 2011: Spiders (Araneida) from slopes of Macošská strán̆ and Vilémovická stráň (Protected landscape area of Moravian carst, Czech Republic) Acta Musei Moravie, Scientiae biologicae, 96(1): 1-27.

NOFLATCHER, M.-T., 1988: Ein Beitrag zur Spinnenfauna Südtirols: Epigäische Spinnen an Xerotherm- und Kulturstandorten bei Albeins. Berichte des naturwissenschaftlichen-medizinischen Verein Innsbruck, 75: 147-170.

ÖBERG, S., MAYR, S., DAUBER, J., 2008: Landscape effects on recolonisation patterns of spiders in arable fields. Agriculture, Ecosystems and Environment, 123: 211-218.

PLATNICK, N. I., 201l: The world spider catalog, version 12.0. American Museum of Natural History. Available online: http://research.amnh. org/iz/spiders/catalog/INTROl.html (cited: 5. 3. 2011)

POTUŽÁKOVÁ, H., 1975: Arachnofauna stepi v Troji a Podhoří. [Spiders of the steppe in Troja and Podhoří]. MSc. thesis, Charles University, Praha, 82 pp.

ROBERTS, M., 1987: Spiders of Great Britain and Ireland, volume II: Linyphiidae and check list. Harley Books, Colchester, 204 pp.

ROBERTS, M., 1995: Spiders of Britain \& Northern Europe. Harper Collins Publishers, London, 383 pp.

RU゚ŽIČKA, V., 2005: Araneae (pavouci), pp. 76-82. In: FARKAČ, J., KRÁL, D., ŠKORPÍK, M. [eds.]: Červený seznam ohrožených druhů České republiky. Bezobratlí. (Red list of threatened species in the Czech Republic. Invertebrates). AOPK ČR, Praha, 760 pp.

RŮŽIČKA, V., BUCHAR, J., 2008: Dodatek ke katalogu pavouků České republiky 2001-2007. Supplement to the Catalogue of Spiders of the Czech Republic 2001-2007. Sborník Oblastního muzea v Mosté, Řada prírodovédná, 29/30:3-32.

ŘEZÁČ, M., PEKÁR, S., KOCOUREK, F., 2006: Effect of Bt-Maize on Epigeic Spiders (Araneae) and Harvestmen (Opiliones). Plant Protection Science, 42(1): 1-8.

ŘEZÁČ, M., 2009: Metodika inventarizace druhů pavouků, pp. 140-153. In: BARTONIČKA, T., (ed.), Metodika inventarizačních průzkumů maloplošných zvláště chráněných území. Dostupné online on: http://www.ochranaprirody. cz/res/data/181/023382.pdf (cited: 5. 3. 2011). 
SLAVÍK, B., 1984: Grundlegende Phytochorotypen der Tschechischen Sozialistischen Republik. Preslia, 56: 241-265.

THALER, K., BUCHAR, J., 1996: Die Wolfspinnen von Österreich 3: Gattungen Aulonia, Pardosa (p. p.), Pirata, Xerolycosa (Arachnida, Araneae: Lycosidae) - Faunistisch-tiergeographische Übersicht. Carinthia II, 183/106: 393-410. ISSN 0374-6771.
TRETZEL, E., 1954: Reife- und Fortpflanzungszeit bei Spinnen. Zeitschrift für Morphologie und Okologie der Tiere, 42: 634-691.

VALEŠOVÁ, E., 1962: Arachnofauna stepní lokality Lochkov-Radotín. [Spiders of the steppe locality Lochkov-Radotín]. MSc. thesis, Charles University, Praha, 128 pp.

\section{Address}

Ing. Vladimír Hula, Ph.D., Ing. Ondřej Košulič, Dr. Ing. Pavla Štastná, Ústav zoologie, rybářství, hydrobiologie a včelařství, Mendelova univerzita v Brně, Zemědělská 1, 61300 Brno, Česká republika, e-mail: hula@ mendelu.cz 
